\title{
Survival of Hepatocellular Carcinoma Patients Treated with Sorafenib beyond Progression
}

\author{
Leonidas Apostolidis ${ }^{a, h} \quad$ Jan Pfeiffenberger ${ }^{b, h} \quad$ Daniel Gotthardt ${ }^{b}, \mathrm{~h}$ \\ Boris Radeleff ${ }^{c, h} \quad$ Arianeb Mehrabi ${ }^{d, h}$ Peter Schemmer ${ }^{d, e, h}$ \\ Dirk Jäger ${ }^{a}, h \quad$ Peter Schirmacher ${ }^{f, h}$ Wolfgang Stremmel ${ }^{b, h}$ \\ Henning Schulze-Bergkamen ${ }^{a}, \mathrm{~g}, \mathrm{~h} \quad$ Christoph Springfeld ${ }^{\mathrm{a}}{ }^{\mathrm{h}}$ \\ Karl Heinz Weiss ${ }^{b}, \mathrm{~h}$ \\ a Department of Medical Oncology, National Center for Tumor Diseases, University Hospital \\ Heidelberg, Heidelberg, Germany; ${ }^{b}$ Department of Internal Medicine IV, University Hospital \\ Heidelberg, Heidelberg, Germany; ' $D$ epartment of Diagnostic and Interventional Radiology, \\ University Hospital Heidelberg, Heidelberg, Germany; dDepartment of General, Visceral \\ and Transplant Surgery, University Hospital Heidelberg, Heidelberg, Germany; ${ }^{\text {Department }}$ \\ of Surgery, Medical University of Graz, Graz, Austria; ${ }^{f}$ Institute of Pathology, University \\ Hospital Heidelberg, Heidelberg, Germany; ${ }^{9}$ Department of Internal Medicine 2, St. Mary

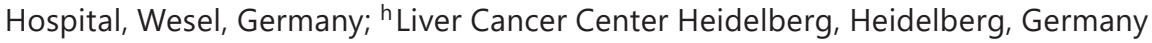

\section{Keywords}

Hepatocellular carcinoma · Molecular-targeted therapies · Therapeutics · Therapy · Treatment · Progression · Sorafenib · Survival

\begin{abstract}
Background/Aim: Sorafenib leads to improved survival in advanced hepatocellular carcinoma (HCC) patients. Continuation of sorafenib beyond progression has been a possible treatment strategy when further approved therapeutic agents are lacking. Methods: We performed a retrospective analysis of all HCC patients at our institution with documented disease progression under treatment with sorafenib. Overall survival (OS) from start of sorafenib treatment was compared between patients who received sorafenib for $>3$ weeks beyond progression (group 1) and those who discontinued sorafenib $\leq 3$ weeks after progression (group 2). Group 1 was further subdivided into those patients who received sorafenib for $>3$ months (group 1a) and those who received it for $\leq 3$ months (group 1b). Results: A total of 71 patients were analyzed. Median OS for all patients was 15.4 months. OS in group 1 (15.6 months) and 2 (13.0 months) was similar ( $p=0.90$ ). Patients in group 1a showed significantly prolonged median OS (19.7 months) compared to that of patients in group $1 \mathrm{~b}$ (13.6 months, $p=0.004$ )
\end{abstract}


and they showed a trend towards prolonged OS compared to group 2 ( $p=0.126)$. For patients with a poor prognosis according to their Child-Pugh stage, performance status, alpha-fetoprotein, and response to prior sorafenib treatment, OS was significantly prolonged in group 1 versus group 2 (12.1 vs. 6.4 months, $p=0.019$ ). Conclusion: In HCC patients, continuing sorafenib beyond progression for $>3$ months is associated with improved survival compared to discontinuing sorafenib within 3 months. Furthermore, patients with a poor prognosis who continue sorafenib beyond progression in general show significantly prolonged survival.

(C) 2018 S. Karger AG, Base

\section{Introduction}

Hepatocellular carcinoma (HCC) is the sixth most common malignancy worldwide and the second leading cause of cancer-related death [1]. For advanced or metastatic HCC, the multi-tyrosine-kinase inhibitor sorafenib has been the only approved first-line systemic therapeutic option so far. It has shown a moderate but significant prolongation of overall survival $(\mathrm{OS})$ versus placebo in 2 large phase III trials [2, 3]. In the absence of systemic therapeutic alternatives, it has been discussed to continue sorafenib treatment in patients despite disease progression. In the SHARP trial, in some patients, treatment was continued until radiological and clinical progression, implying that several patients were treated beyond radiological progression as long as there was no evidence of clinical progression [2]. However, details concerning the outcome of this patient group are lacking. Several retrospective reports suggest a benefit of continuing sorafenib treatment beyond progression in HCC patients [4-7]. For other tumor types, there is evidence that to continue a targeted therapy beyond progression may lead to an improved outcome. For example, this has been shown for bevacizumab in colorectal cancer patients [8-10] and trastuzumab in breast cancer patients [11]. In lung cancer patients, discontinuation of a targeted therapy has been reported to result in a disease flare-up and even worse prognosis [12-15].

The aim of our study was to investigate the outcome of patients under sorafenib treatment who continued this treatment despite disease progression and to compare the results with those of patients who discontinued treatment after progression.

\section{Patients and Methods}

We retrospectively reviewed the medical records of all patients under sorafenib therapy for HCC at the University Hospital Heidelberg, Germany, with documented radiological progression between November 2011 and July 2015. All patients provided written informed consent before enrolment in the study. Prior, the study design was approved by the Institutional Research Ethics Committee.

We analyzed the patients' demographic characteristics, course of disease under sorafenib treatment, as well as dose modifications, and treatment interruptions. The patients who discontinued sorafenib due to toxicity without documented disease progression, who received liver transplantation prior to sorafenib therapy, or to whom sorafenib was administered as a "bridge to transplant" were excluded from the study.

HCC was diagnosed according to the current American Association for the Study of Liver Diseases guidelines [16]. Each case was evaluated by an interdisciplinary liver tumor board conference. Only those patients with no curative therapeutic options and contraindications for locoregional therapies received sorafenib treatment. Concomitant medication was prescribed in case of adverse events. The standard initial dose of sorafenib was $400 \mathrm{mg}$ twice daily. The dose was adjusted in the case of adverse events that were not manageable by supportive care. The patients were seen at our outpatient clinic every 2-3 months.

Radiological assessment was done every 3 months using contrast-enhanced MRI, or, in cases with contraindications, using contrast-enhanced CT. Radiographic assessment was performed according to the Response Evaluation Criteria in Solid Tumors (RECIST) version 1.1. Upon radiographically proven disease progression under sorafenib, the patients were offered to continue the treatment if their clinical condition 
was adequate and no other treatment options - like participation in a clinical trial - were available. The decision whether to continue or to stop sorafenib after progression was made by the treating physician who took the patient's choice into account. Treatment was stopped in the event of deterioration of the clinical status or unmanageable adverse events. OS was defined as the time between the initiation of sorafenib treatment and death of any cause. Time to progression was defined as the time between initiation of sorafenib treatment and disease progression. For the analysis of treatment beyond progression, 3 groups were defined: patients who continued sorafenib for $>3$ months after progression (group 1a); patients who continued sorafenib for $>3$ weeks but $\leq 3$ months after progression (group $1 \mathrm{~b}$ ); and patients who discontinued sorafenib within 3 weeks after disease progression (group 2).

Statistical analysis was carried out using SPSS ${ }^{\mathrm{TM}}$ for Windows ${ }^{\mathrm{TM}}$ Software v22.0 (SPSS, Chicago, IL, USA). Frequencies were compared with the $\chi^{2}$ test, median values with one-way analysis of variance. Survival analysis was calculated using the Kaplan-Meier method, and differences in survival were analyzed using the log-rank test. A $p$ value $<0.05$ was considered significant.

\section{Results}

\section{Baseline Characteristics}

A total of 71 patients were included in the study. Median age was 62 years, $85.9 \%$ of the patients were male (Table 1). $74.6 \%$ of the patients suffered from liver cirrhosis. The most common etiologies for the underlying liver disease were alcohol, chronic hepatitis $\mathrm{C}$, and chronic hepatitis B. Extrahepatic tumor manifestations were present in $43.7 \%$ of the patients. The majority of the patients were at stage $\mathrm{C}$ according to the Barcelona Liver Cancer Classification (BCLC).

\section{Sorafenib Treatment}

$50.7 \%$ of the patients required a dose reduction due to toxicities, treatment had to be interrupted in 29 patients (40.8\%). The responses to standard sorafenib treatment were: partial remission in 1 patient (1.4\%), stable disease in 17 patients $(23.9 \%)$, and progressive disease in 53 patients (74.6\%). Median time to progression was 3.0 months ( $95 \%$ CI 2.7-3.4). Mean daily sorafenib dose was $760 \mathrm{mg}$ with no significant difference between the treatment groups. After progression under sorafenib treatment, 27 patients (38.0\%) were discontinued within 3 weeks (group 2). Of the remaining patients (group 1), 19 patients (26.8\%) continued sorafenib for $\leq 3$ months (group $1 \mathrm{~b}$ ), 25 patients (35.2\%) continued the treatment for $>3$ months (group 1a). There were no significant differences between the treatment groups for most patient characteristics; however, the frequency of esophageal varices was significantly higher in group 2, and the proportion of patients pretreated with transarterial chemoembolization was significantly lower in this group (Table 1). Subsequent treatments after sorafenib included chemotherapy in $8.5 \%$ of the patients and participation in a clinical trial for nonapproved substances in $22.5 \%$ of the patients (group 1a: $36.0 \%$, group 1b: $5.2 \%$, group 2: 22.2\%). Clinical trial treatment included the HDAC inhibitor resminostat (ClinicalTrials.gov identifier NCT00943449), the anti-glypican3 antibody GC33 (NCT01507168), the recombinant vaccinia virus JX-594 (NCT01387555), the MET inhibitor tivantinib (NCT01755767), and the PD-L1 antibody durvalumab (NCT01693562). No patient received regorafenib.

\section{Survival}

Median OS for all patients was 15.4 months (95\% CI 12.8-18.1). Patients who continued sorafenib well beyond progression (group 1) showed a median OS of 15.6 months (95\% CI 13.3-17.9) compared to those patients who discontinued the treatment within 3 weeks after progression (group 2; 13.0 months, 95\% CI 0.7-25.2, $p=0.90$ ) (Fig. 1). Patients who received sorafenib for $>3$ months beyond progression (group 1a) showed significantly prolonged 
Apostolidis et al.: Sorafenib beyond Progression for HCC

Table 1. Patient characteristics at the start of sorafenib treatment and at disease progression under sorafenib

\begin{tabular}{|c|c|c|c|c|c|}
\hline & All patients $(n=71)$ & Group 1a $(n=25)$ & Group 1b $(n=19)$ & Group $2(n=27)$ & $p$ \\
\hline \multicolumn{6}{|c|}{ At the start of sorafenib treatment } \\
\hline \multicolumn{6}{|c|}{ Age, years } \\
\hline Median & 62 & 66 & 62 & 62 & \multirow[t]{2}{*}{0.682} \\
\hline Range & $26-84$ & $32-84$ & $32-76$ & $26-80$ & \\
\hline \multicolumn{6}{|l|}{ Sex } \\
\hline Male & $61(85.9)$ & $24(96.0)$ & 17 (89.5) & $20(74.1)$ & \multirow[t]{2}{*}{0.066} \\
\hline Female & $10(14.1)$ & $1(4.0)$ & $2(10.5)$ & 7 (25.9) & \\
\hline \multicolumn{6}{|l|}{ ECOG } \\
\hline 0 & $49(69.0)$ & $19(76.0)$ & $12(63.2)$ & $18(66.7)$ & \multirow[t]{3}{*}{0.372} \\
\hline 1 & $20(28.2)$ & $6(24.0)$ & 7 (36.8) & 7 (25.9) & \\
\hline 2 & $2(2.8)$ & 0 & 0 & $2(7.4)$ & \\
\hline \multicolumn{6}{|l|}{ BCLC stage } \\
\hline A & $3(4.2)$ & $2(8.0)$ & 0 & $1(3.7)$ & \multirow[t]{3}{*}{0.080} \\
\hline B & $22(31.0)$ & $6(24.0)$ & $3(15.8)$ & $13(48.1)$ & \\
\hline $\mathrm{C}$ & $46(64.8)$ & $17(68.0)$ & $16(84.2)$ & $13(48.1)$ & \\
\hline \multicolumn{6}{|l|}{ Child-Pugh stage } \\
\hline A & 58 (81.7) & $23(92.0)$ & $14(73.7)$ & $21(77.8)$ & \multirow[t]{2}{*}{0.238} \\
\hline B & $13(18.3)$ & $2(8.0)$ & $5(26.3)$ & $6(22.2)$ & \\
\hline Liver cirrhosis & $53(74.6)$ & $18(72.0)$ & $15(78.9)$ & $20(74.1)$ & 0.868 \\
\hline \multicolumn{6}{|l|}{ Etiology } \\
\hline Alcohol & $25(35.2)$ & $9(36.0)$ & $7(36.8)$ & $9(33.3)$ & 0.965 \\
\hline Hepatitis B & $15(21.1)$ & $2(8.0)$ & $4(21.1)$ & $9(33.3)$ & 0.082 \\
\hline Hepatitis C & $18(25.4)$ & $10(40.0)$ & $4(21.1)$ & $4(14.8)$ & 0.100 \\
\hline Cryptogenic & $6(8.5)$ & 0 & $3(15.8)$ & $3(11.1)$ & 0.144 \\
\hline NASH & $4(5.6)$ & $1(4.0)$ & $1(5.3)$ & $2(7.4)$ & 0.865 \\
\hline Ascites & $38(53.5)$ & $11(44.0)$ & $11(57.9)$ & $16(59.3)$ & 0.493 \\
\hline Extrahepatic manifestations & $31(43.7)$ & $14(56.0)$ & $9(47.4)$ & $11(40.7)$ & 0.545 \\
\hline Portal vein thrombosis & $16(22.5)$ & 7 (28.0) & $3(15.8)$ & $6(22.2)$ & 0.789 \\
\hline Esophageal varices & $23(32.4)$ & $5(20.0)$ & $5(26.3)$ & $14(51.9)$ & 0.038 \\
\hline \multicolumn{6}{|l|}{ Prior HCC therapy } \\
\hline TACE & $28(39.4)$ & $11(44.0)$ & $11(57.9)$ & $6(22.2)$ & 0.043 \\
\hline Resection & $22(31.0)$ & 7 (28.0) & $4(21.1)$ & $11(40.7)$ & 0.336 \\
\hline RFA & $6(8.5)$ & $2(8.0)$ & $2(10.5)$ & $2(7.4)$ & 0.928 \\
\hline SIRT & $15(21.1)$ & $2(8.0)$ & $4(21.1)$ & $9(33.3)$ & 0.082 \\
\hline \multicolumn{6}{|l|}{ At disease progression } \\
\hline \multicolumn{6}{|l|}{ ECOG } \\
\hline 0 & $44(62.0)$ & $16(64.0)$ & $12(63.2)$ & $16(59.3)$ & \multirow[t]{4}{*}{0.551} \\
\hline 1 & $21(29.6)$ & $9(36.0)$ & $5(26.3)$ & 7 (25.9) & \\
\hline 2 & $5(7.0)$ & 0 & $2(10.5)$ & $3(11.1)$ & \\
\hline 3 & $1(1.4)$ & 0 & 0 & $1(3.7)$ & \\
\hline \multicolumn{6}{|l|}{ Child-Pugh stage } \\
\hline A & $54(76.1)$ & $20(80.0)$ & $14(73.7)$ & $20(74.1)$ & \multirow[t]{3}{*}{0.709} \\
\hline B & $14(19.7)$ & $4(16.0)$ & $5(26.3)$ & 5 (18.5) & \\
\hline C & $3(4.2)$ & $1(4.0)$ & 0 & $2(7.4)$ & \\
\hline \multicolumn{6}{|l|}{ Best response under sorafenib } \\
\hline $\mathrm{PR}$ & $1(1.4)$ & 0 & $1(5.3)$ & 0 & \multirow[t]{3}{*}{0.153} \\
\hline SD & 17 (23.9) & $4(16.0)$ & $3(15.8)$ & $10(37.0)$ & \\
\hline PD & 53 (74.6) & $21(84.0)$ & 15 (78.9) & $17(63.0)$ & \\
\hline $\mathrm{AFP}, \mathrm{ng} / \mathrm{mL}$ & & & & & \\
\hline Median & 59 & 58 & 362 & 8.3 & 0.429 \\
\hline$>1,000$ & $22(31.0)$ & $6(24.0)$ & 7 (36.8) & $9(33.3)$ & 0.623 \\
\hline
\end{tabular}

Unless indicated otherwise, values are $n$ with percentages in parentheses. Group 1a: continued sorafenib for $>3$ months, group $1 \mathrm{~b}$ : continued sorafenib for $>3$ weeks but $\leq 3$ months, group 2: discontinued sorafenib within 3 weeks. AFP, alphafetoprotein; BCLC, Barcelona Classification for Liver Cancer; ECOG, Eastern Cooperative Oncology Group performance status; NASH, non-alcoholic steatohepatitis; PD, progressive disease; PR, partial remission; RFA, radio-frequency ablation; SD, stable disease; SIRT, selective internal radiotherapy; TACE, transarterial chemoembolization. 
Fig. 1. Overall survival of patients in group 1 (continuing sorafenib beyond progression) versus group 2 (discontinuing sorafenib after progression).

Fig. 2. Overall survival of patients in group 1a (continuing sorafenib beyond progression for $>3$ months) versus group $1 \mathrm{~b}$ (continuing sorafenib beyond progression for $\leq 3$ months).

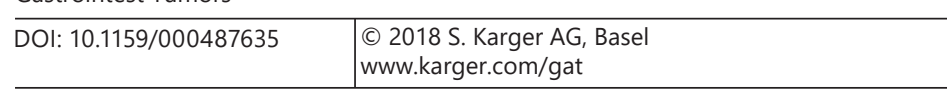

Apostolidis et al.: Sorafenib beyond Progression for HCC
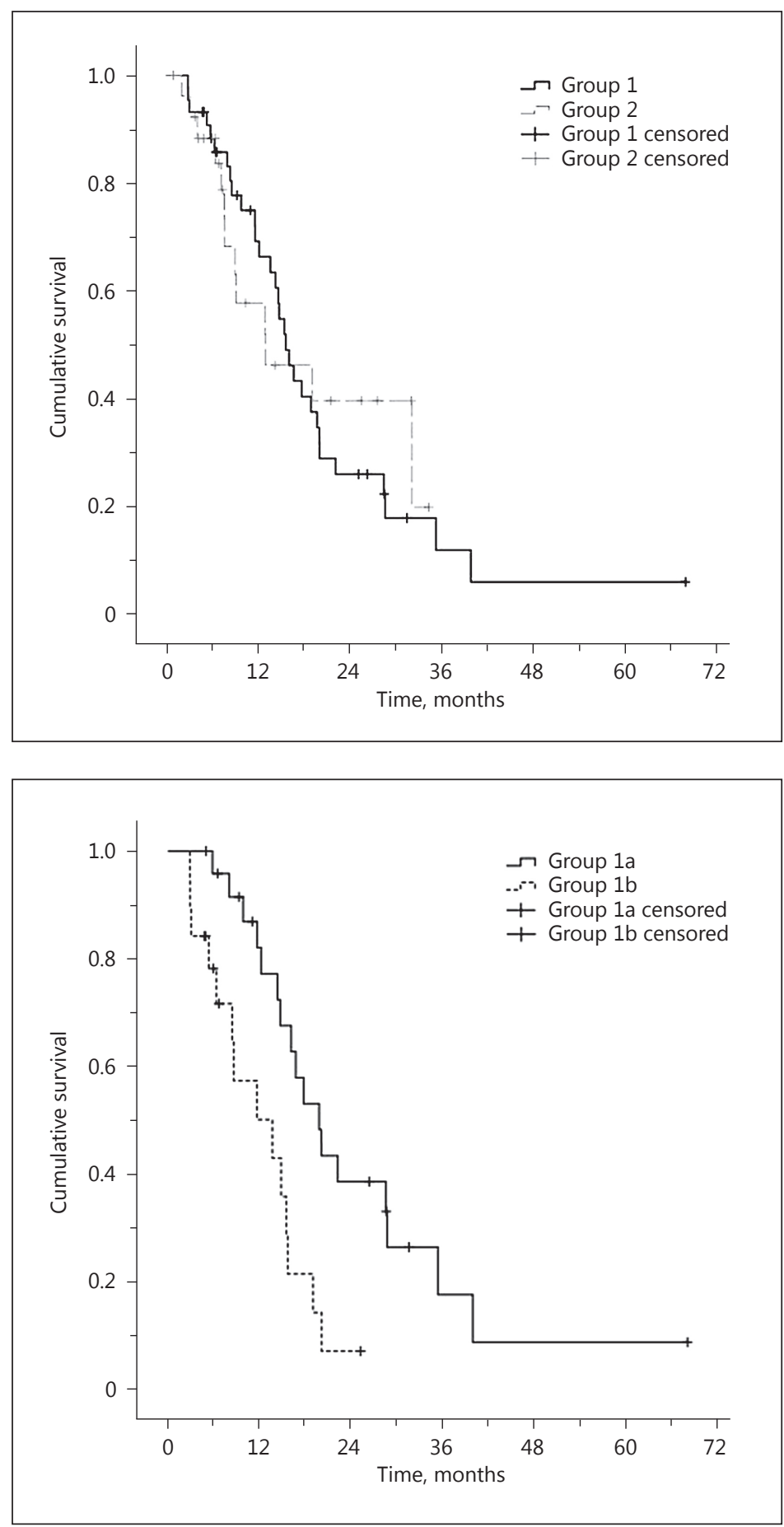

median OS (19.7 months, 95\% CI 14.8-24.7) in comparison to those who continued treatment for $\leq 3$ months (group 1b; 13.6 months, 95\% CI 4.8-22.4, $p=0.004$ ) (Fig. 2), and they also showed a trend towards prolonged OS compared to the patients of group 2 ( $p=0.126$ ) (see online suppl. Fig. 1; see www.karger.com/doi/10.1159/000487635 for all online suppl. material). A stronger trend could be observed in comparison to groups $1 \mathrm{~b}$ and 2 combined (13.0 months, 95\% CI 7.1-18.8, $p=0.062$ ) (see online suppl. Fig. 2). 
Fig. 3. Overall survival of group $1 \mathrm{a}$ versus group $1 \mathrm{~b}$ patients with BCLC stage C.

\section{DOI: 10.1159/000487635 \\ (c) 2018 S. Karger AG, Base \\ www.karger.com/gat}

Apostolidis et al.: Sorafenib beyond Progression for HCC

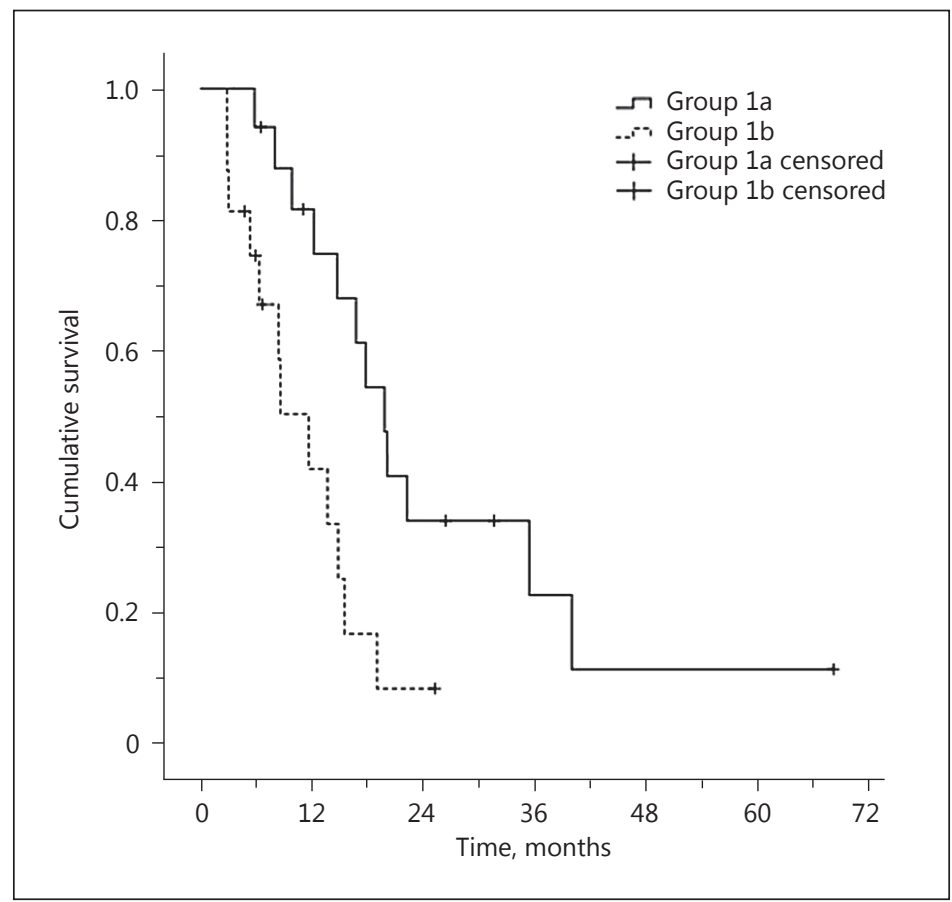

When looking at the 46 BCLC stage C patients, OS was significantly prolonged in the 17 group 1a patients (19.7 months, 95\% CI 15.6-23.9) versus the 16 group $1 \mathrm{~b}$ patients $(11.6$ months, 95\% CI 6.4-16.8, $p=0.010$ ) (Fig. 3). Excluding patients treated in clinical trials from the analysis resulted in a median OS of 16.1 months for group 1a (95\% CI 9.7-22.5), 11.6 months for group $1 \mathrm{~b}(95 \% \mathrm{CI} 5.7-17.4, p=0.164)$, and 9.1 months for group 2 (95\% CI 2.5-15.8, $p=0.737$ ) (see online suppl. Fig. 3).

Additionally, we evaluated a prognostic scoring system for post-progression OS according to the study by Otsuka et al. [17], with poor prognosis being defined by a Child-Pugh stage C or at least 2 of the following characteristics: Child-Pugh stage B, ECOG performance status $>1$, progressive disease as best response to sorafenib, or alpha-fetoprotein $>1,000 \mathrm{ng} / \mathrm{mL}$. Patients with a poor prognosis in group 1 showed significantly prolonged OS (12.1 months, 95\% CI 7.8-16.4) versus those with a poor prognosis in group 2 (6.4 months, 95\% CI 2.9-9.9, $p=0.019)$. However, OS for patients with a good prognosis showed no significant difference between group 1 and 2 (20.0 vs. 32.1 months, $p=0.814$ ) (Fig. 4).

\section{Discussion}

The idea to continue targeted systemic therapy beyond radiographic progression has been examined in several tumor types. Improved prognosis of patients continuously treated has be observed in colorectal [8-10], breast [11], and lung cancer [12-15]. Several mechanisms have been proposed for this phenomenon, including an accumulation of upstream products of the inhibited signaling pathways as well as an activation of alternative signaling pathways [6]; in those cases, a rapid discontinuation of the targeted therapy might result in an overproportional tumor growth stimulus.

In HCC patients, with sorafenib having been the only approved therapeutic agent for a long time, treatment beyond progression has been discussed as a therapeutic option. In our study, we 
Fig. 4. Overall survival of patients with a poor or a good prognosis according to Otsuka et al. [17] in groups 1 and 2 .

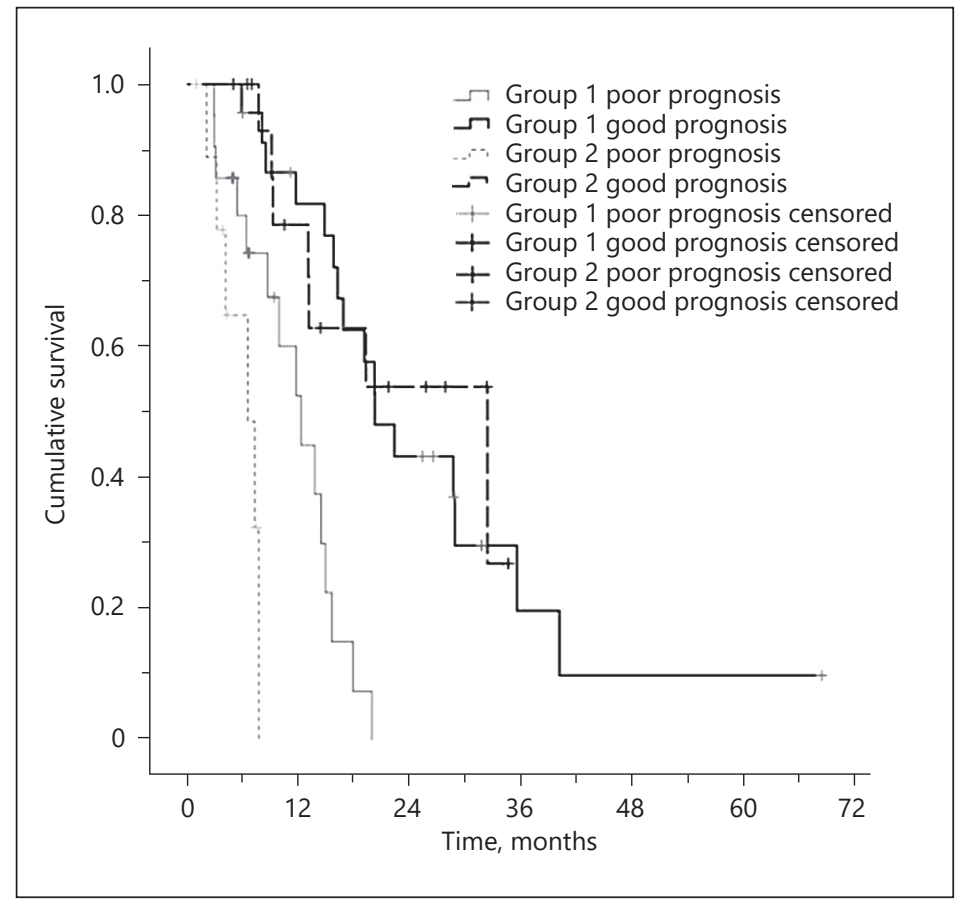

could show a significantly longer survival of patients treated with sorafenib beyond progression for $>3$ months compared to that of patients treated for $<3$ months, as well as a trend towards a prolongation of survival compared to patients who discontinued sorafenib. The effect was most notable in the subgroup of BCLC stage $C$ patients, with a median survival benefit of 8.1 months.

The recently presented preliminary data of the RESOURCE trial demonstrate an improved OS of 10.6 versus 7.8 months for regorafenib versus placebo after progression under sorafenib [18]. Since regorafenib has a similar kinase-inhibiting activity as sorafenib, and since the patients in the RESOURCE trial had tolerated the preceding sorafenib treatment well, it can be argued that an OS benefit similar to that achieved with regorafenib could have been achieved had sorafenib been continued beyond progression.

On the other hand, so far there are no prospective randomized trials which clearly show a benefit of continued sorafenib treatment beyond progression for HCC patients. A prospective phase II trial comparing dose increase of sorafenib after progression to best supportive care showed only a trend towards a prolongation of OS and progression-free survival, but it failed to reach statistical significance [19]. In this trial, however, toxicity due to dose escalation of sorafenib as well as randomization imbalances might have negatively affected the outcome of patients treated beyond progression.

Our study has several limitations since it was carried out retrospectively. The decision whether or not to continue sorafenib beyond progression was made by the treating physician who took the patient's choice into account. Therefore, the observed survival benefit could just reflect the natural course of the disease after progression, and those patients with prolonged survival due to a more favorable tumor biology could receive sorafenib treatment for a longer time, meaning that prolonged survival was the cause of the longer sorafenib treatment and not vice versa. However, there were no significant differences for most patient characteristics between the treatment groups, with the exception of a higher proportion of esophageal varices and a lower proportion of transarterial chemoembolization pretreatment in those patients who discontinued sorafenib within 3 weeks after progression. 
Furthermore, the results of our analysis support those of several other previous retrospective studies. In an analysis of 43 patients, Woo et al. [7] could show a prolongation of median survival in patients treated with sorafenib beyond progression versus best supportive care (81 vs. 131 days). However, a higher survival rate could be observed in patients receiving additional locoregional treatment or an alternate therapy without sorafenib (e.g., in a clinical trial). In another study on 88 patients progressing under sorafenib, the median OS was prolonged in patients continuing on sorafenib (355 vs. 517 days), and $12.5 \%$ of the patients discontinuing sorafenib suffered disease outbreaks [6]. In an analysis of 36 patients, Miyahara et al. [5] could show a significant increase in the tumor growth rate as well as shorter survival in those patients who discontinued sorafenib (8.4 vs. 15.0 months). In a retrospective study of 101 patients, a scoring system including Child-Pugh stage, ECOG, best response to sorafenib, and alpha-fetoprotein levels could identify a patient subpopulation which showed significantly prolonged post-progression survival when continued on sorafenib or other treatments versus best supportive care [17]. Improved survival could be detected in those patients with a favorable prognostic score. Surprisingly, when applying this risk score to our analysis, a significantly improved OS under sorafenib could only be detected in those patients with a poor prognosis. This might be due to different baseline characteristics of both studies as well as the fact that Otsuka et al. [17] combined both sorafenib and other treatments into one group in their study. A prognostic validation of this score might clarify its role in treatment decision.

In addition to the results of those previous reports, our study demonstrated that not only to continue sorafenib treatment beyond progression per se is associated with improved OS, but also the duration of this treatment for $>3$ months.

Despite recent advances in systemic therapy with regorafenib [18], MET inhibition [20], and immune checkpoint blockade [21], sorafenib still remains the only approved first-line systemic treatment option for patients with advanced HCC with proven prolongation of OS versus placebo. Further effective systemic therapies after progression under sorafenib are still desperately needed. In our opinion, the data of our study as well as the cited literature suggest that HCC patients with progressing disease under sorafenib treatment who are not suitable for a clinical trial could be offered a continuation of sorafenib. Further studies are needed to select the right patients for treatment beyond progression as well as to explore sorafenib as an option after failure of second-line therapy.

\section{Statement of Ethics}

All patients provided written informed consent before enrolment in the study. Prior, the study design was approved by the Institutional Research Ethics Committee.

\section{Disclosure Statement}

Karl Heinz Weiss has received fees for serving as a speaker and he is an advisory board member for Bayer. Additionally, he has received research funding from Bayer. All other authors have no conflicts of interest to declare. 


\section{References}

1 Torre LA, Bray F, Siegel RL, Ferlay J, Lortet-Tieulent J, Jemal A: Global cancer statistics, 2012. CA Cancer J Clin 2015;65:87-108.

-2 Llovet JM, Ricci S, Mazzaferro V, et al: Sorafenib in advanced hepatocellular carcinoma. N Engl J Med 2008;359: 378-390.

-3 Cheng AL, Kang YK, Chen Z, Tsao CJ, Qin S, Kim JS, Luo R, Feng J, Ye S, Yang TS, Xu J, Sun Y, Liang H, Liu J, Wang J, Tak WY, Pan H, Burock K, Zou J, Voliotis D, Guan Z: Efficacy and safety of sorafenib in patients in the AsiaPacific region with advanced hepatocellular carcinoma: a phase III randomised, double-blind, placebocontrolled trial. Lancet Oncol 2009;10:25-34.

4 Reig M, Rimola J, Torres F, Darnell A, Rodriguez-Lope C, Forner A, Llarch N, Rios J, Ayuso C, Bruix J: Postprogression survival of patients with advanced hepatocellular carcinoma: rationale for second-line trial design. Hepatology 2013;58:2023-2031.

5 Miyahara K, Nouso K, Morimoto Y, Takeuchi Y, Hagihara H, Kuwaki K, Onishi H, Ikeda F, Miyake Y, Nakamura S, Shiraha H, Takaki A, Iwadou S, Kobayashi Y, Takaguchi K, Takuma Y, Takabatake H, Sakaguchi K, Yamamoto K; Okayama Liver Cancer Group: Efficacy of sorafenib beyond first progression in patients with metastatic hepatocellular carcinoma. Hepatol Res 2014;44:296-301.

-6 Fu SR, Zhang YQ, Li Y, Hu BS, He X, Huang JW, Zhan MX, Lu LG, Li JP: Sorafenib continuation after first disease progression could reduce disease flares and provide survival benefits in patients with hepatocellular carcinoma: a pilot retrospective study. Asian Pac J Cancer Prev 2014;15:3151-3156.

7 Woo HY, Heo J, Yoon KT, Kim GH, Kang DH, Song GA, Cho M: Clinical course of sorafenib treatment in patients with hepatocellular carcinoma. Scand J Gastroenterol 2012;47:809-819.

-8 Bennouna J, Sastre J, Arnold D, Osterlund P, Greil R, Van Cutsem E, von Moos R, Vieitez JM, Bouche O, Borg C, Steffens CC, Alonso-Orduna V, Schlichting C, Reyes-Rivera I, Bendahmane B, Andre T, Kubicka S; ML18147 Study Investigators: Continuation of bevacizumab after first progression in metastatic colorectal cancer (ml18147): a randomised phase 3 trial. Lancet Oncol 2013;14:29-37.

-9 Grothey A, Flick ED, Cohn AL, Bekaii-Saab TS, Bendell JC, Kozloff M, Roach N, Mun Y, Fish S, Hurwitz HI: Bevacizumab exposure beyond first disease progression in patients with metastatic colorectal cancer: analyses of the ARIES observational cohort study. Pharmacoepidemiol Drug Saf 2014;23:726-734.

10 Grothey A, Sugrue MM, Purdie DM, Dong W, Sargent D, Hedrick E, Kozloff M: Bevacizumab beyond first progression is associated with prolonged overall survival in metastatic colorectal cancer: results from a large observational cohort study (BRiTE). J Clin Oncol 2008;26:5326-5334.

11 Petrelli F, Barni S: A pooled analysis of 2,618 patients treated with trastuzumab beyond progression for advanced breast cancer. Clin Breast Cancer 2013;13:81-87.

$\checkmark 12$ Chaft JE, Oxnard GR, Sima CS, Kris MG, Miller VA, Riely GJ: Disease flare after tyrosine kinase inhibitor discontinuation in patients with EGFR-mutant lung cancer and acquired resistance to erlotinib or gefitinib: implications for clinical trial design. Clin Cancer Res 2011;17:6298-6303.

13 Pop 0, Pirvu A, Toffart AC, Moro-Sibilot D: Disease flare after treatment discontinuation in a patient with EML4-ALK lung cancer and acquired resistance to crizotinib. J Thorac Oncol 2012;7:e1-e2.

14 Yang JJ, Chen HJ, Yan HH, Zhang XC, Zhou Q, Su J, Wang Z, Xu CR, Huang YS, Wang BC, Yang XN, Zhong WZ, Nie Q, Liao RQ, Jiang BY, Dong S, Wu YL: Clinical modes of EGFR tyrosine kinase inhibitor failure and subsequent management in advanced non-small cell lung cancer. Lung Cancer 2013;79:33-39.

15 Chen HJ, Yan HH, Yang JJ, Chen ZH, Su J, Zhang XC, Wu YL: Disease flare after EGFR tyrosine kinase inhibitor cessation predicts poor survival in patients with non-small cell lung cancer. Pathol Oncol Res 2013;19:833838.

16 Bruix J, Sherman M: Management of hepatocellular carcinoma. Hepatology 2005;42:1208-1236.

17 Otsuka T, Nakashita S, Yanagita K, Ario K, Kawasoe H, Kawazoe S, Eguchi Y, Mizuta T: Factors associated with post-progression survival in patients with advanced hepatocellular carcinoma treated with sorafenib. Diseases 2015;3:68-77.

18 Bruix J, Qin S, Merle P, et al: Regorafenib for patients with hepatocellular carcinoma who progressed on sorafenib treatment (RESORCE): a randomised, double-blind, placebo-controlled, phase 3 trial. Lancet 2017; 389:56-66.

19 Rimassa L, Pressiani T, Boni C, Carnaghi C, Rota Caremoli E, Fagiuoli S, Foa P, Salvagni S, Cortesi E, Chiara Tronconi M, Personeni N, Bozzarelli S, Chiara Banzi M, Fanello S, Romano Lutman F, Giordano L, Santoro A: A phase II randomized dose escalation trial of sorafenib in patients with advanced hepatocellular carcinoma. Oncologist 2013;18:379-380.

20 Santoro A, Rimassa L, Borbath I, et al: Tivantinib for second-line treatment of advanced hepatocellular carcinoma: a randomised, placebo-controlled phase 2 study. Lancet Oncol 2013;14:55-63.

21 El-Khoueiry AB, Melero I, Crocenzi TS, Welling TH, Yau TC, Yeo W, Chopra A, Grosso J, Lang L, Anderson J, Cruz CMD, Sangro B: Phase I/II safety and antitumor activity of nivolumab in patients with advanced hepatocellular carcinoma (HCC): Ca209-040. J Clin Oncol 2015;33(suppl):abstr LBA101. 